\title{
Autoimmune-like Drug-induced Liver Injury Caused by Atorvastatin and Demonstration of the Safety Profile of Pravastatin: A Case Report and Literature Review
}

\author{
Adeel A. Khan ${ }^{1}$, Salma Ahmed ${ }^{2}$, Abdul Mohammed ${ }^{3}$, Abdel-Naser Y. Elzouki ${ }^{1,4}$ \\ 1. Internal Medicine, Hamad Medical Corporation, Doha, QAT 2. Community Medicine, Hamad Medical Corporation, \\ Doha, QAT 3. General Medicine, Hamad Medical Corporation, Doha, QAT 4. Medicine, Weill Cornell Medical College, \\ Doha, QAT
}

Corresponding author: Adeel A. Khan, adeel_1026@yahoo.com

\begin{abstract}
Statin-induced liver injury is a well-recognized but rare phenomenon with hepatocellular, cholestatic, and mixed phenotypes. Most studies do not recommend regular monitoring of liver function tests (LFTs) after starting statins unless clinically indicated. We report a case of autoimmune-like atorvastatin-induced liver injury (aminotransferases $>5$ times the upper limit of normal) that was detected on routine follow-up after three months in an asymptomatic patient. In addition to elevation in transaminases, the patient had weakly positive ANAs. Anti-smooth muscle antibody (ASMA) was positive in titers of 1:680. Screening for viral hepatitis A-E was negative. Other diagnostic investigations showed complete blood examination, including eosinophils, renal function tests, electrolytes, total protein, albumin, prothrombin time, activated partial thromboplastin time (aPTT), international normalized ratio (INR), serum ferritin, and iron saturation to be in the normal range. Ultrasound and computed tomography (CT) abdomen showed normal liver, gall bladder, biliary tree, and pancreas. The patient was managed as a case of autoimmune-like drug-induced liver injury (DILI) caused by atorvastatin and the medication was discontinued. LFTs returned to completely normal 30 days after the discontinuation of atorvastatin. Furthermore, switching to pravastatin for dyslipidemia management four months after stopping atorvastatin did not lead to hepatotoxicity, illustrating the safety profile of pravastatin in patients who are unable to tolerate atorvastatin.
\end{abstract}

Categories: Internal Medicine, Gastroenterology

Keywords: drug induced liver injury, atorvastatin, pravastatin, autoimmune like

\section{Introduction}

Statins, also known as 3-hydroxy-3methylglutaryl coenzyme A (HMG CoA) reductase inhibitors, are the most commonly used agents for the management of dyslipidemia and cardiovascular risk reduction.

Hepatotoxicity is one of its most feared side-effects. Although asymptomatic and mild elevation of aminotransferases (AST and ALT) is common, a significant elevation in these enzymes is rare and has been reported in less than $3 \%$ cases only [1-2]. Various studies have recommended against the regular monitoring of liver function tests (LFTs) after initiating statins [3-4]. We report a case of atorvastatin related autoimmune-like drug-induced liver injury (DILI) with elevation in aminotransferases > five times the upper limit of normal. It was detected on routine follow-up monitoring in an asymptomatic patient. Furthermore, the use of pravastatin after LFT normalization showed no cross-toxicity with atorvastatin.

\section{Case Presentation}

A 57-year-old female, with a history of dyslipidemia for three to four years, not controlled on dietary and lifestyle modifications, was started on atorvastatin $40 \mathrm{mg}$ once daily. Baseline LFTs were normal. The patient underwent routine LFT testing and had high total and direct bilirubin (122.8 micromol/1 and 85.1 micromol/1 respectively), high alkaline phosphatase (435 U/l), alanine aminotransferase (3195 U/1) and aspartate aminotransferase (2999 U/1) at three months follow-up. The patient was called to report immediately to the emergency department for further investigations. The patient's family noticed yellowish discoloration in her eyes in the last one to two days. The patient also complained of mild low-grade fever three days ago that resolved on its own but didn't complain of chest pain, shortness of breath, sputum production, sore throat, runny nose, abdominal discomfort, itching, vomiting, rash, or change in color of urine. The patient didn’t have any history of alcohol, paracetamol, herbal medication, or any other medication intake. There was no personal or family history of autoimmune diseases or liver diseases. The patient did not have a history of allergy to any medication. On examination, the patient was afebrile, vitally stable, conscious, alert, and had mild scleral jaundice with no stigmata of chronic liver disease. Abdominal examination was unremarkable except for mild tenderness in the epigastric and right upper quadrant regions. Respiratory, cardiac, and nervous system examination was unremarkable. In addition to the abovementioned LFTs results, other laboratory investigations showed normal complete blood examination 


\section{Cureus}

(including normal neutrophil count and eosinophil count), renal function tests, electrolytes, total protein, albumin, prothrombin time, aPTT, INR, serum ferritin, and iron saturation. Urine dipstick was normal. Except for borderline triglyceride of $2.2 \mathrm{mmol} / \mathrm{l}$ (normal $<1.7 \mathrm{mmol} / \mathrm{l}$ ), the lipid profile was within the desirable range. Screening for viral hepatitis A, B, C, and E was negative. She had a weakly positive ANA and positive anti-smooth muscle antibody (ASMA) (1:640). Anti-liver-kidney microsomes, anti-mitochondrial antibody, and screen for connective tissue diseases were negative. Serum immunoglobulin G (IgG) levels were within the normal range $(1210 \mathrm{mg} / \mathrm{dl})$. Ultrasound and CT abdomen showed normal liver, gall bladder, biliary tree, and pancreas (Table 1 ).

\begin{tabular}{|c|c|}
\hline Age (years) & 57 \\
\hline Gender & Female \\
\hline Peak Total Bilirubin (3.4-20.5 micromol/L) & 90.1 \\
\hline Peak Direct bilirubin (0.0-8.6 micromol/L) & 60.3 \\
\hline Peak ALT (Alanine Aminotransferase, Normal = 0-55 U/L) & 3756 \\
\hline Peak AST (Aspartate Aminotransferase, Normal = 5-34 U/L) & 3172 \\
\hline Peak Alkaline Phosphatase (40-150 U/L) & 397 \\
\hline Viral Hepatitis Serology & Negative \\
\hline Time of Onset of Elevation in LFTs (Liver Function Tests) & Within 3 months \\
\hline Time of Return of LFTs (Liver Function Tests) to Normal & Within 30 days \\
\hline RUCAM (Roussel-Uclaf Causality Assessment Method) Score & 9 (highly probable) \\
\hline
\end{tabular}

TABLE 1: Baseline characteristics

The patient was advised liver biopsy to rule out autoimmune hepatitis based on positive autoimmune antibody results but refused and preferred to monitor LFTs on a regular basis. The patient was managed as a case of atorvastatin-induced autoimmune-like DILI. Atorvastatin was discontinued and the patient was followed up in the outpatient clinic with regular monitoring of her LFTs. Twenty-four days after stopping atorvastatin, LFTs improved, indicating drug-induced etiology of elevation in liver enzymes. Total bilirubin became 32.1 micromol/1, alkaline phosphatase normalized at $121 \mathrm{U} / 1$, ALT $211 \mathrm{U} / 1$, and AST 88

$\mathrm{U} / \mathrm{l}$. Aminotransferases returned to normal after one month. After stopping atorvastatin, serum ASMA levels decreased to $1: 80$ in titers.

Follow-up of the patient's lipid profile three months after stopping atorvastatin showed very high total cholesterol (7.2 mmol/l) and LDL (5.58 mmol/l) levels. Pravastatin was introduced and follow up with LFTs was done for 18 months, which showed no elevation in aminotransferases on pravastatin (Table 2). 


\begin{tabular}{|c|c|c|c|c|c|c|c|c|}
\hline & Baseline & $\begin{array}{l}\text { At } \\
\text { diagnosis }\end{array}$ & $\begin{array}{l}24 \text { days after } \\
\text { stopping } \\
\text { atorvastatin }\end{array}$ & $\begin{array}{l}30 \text { days after } \\
\text { stopping } \\
\text { atorvastatin }\end{array}$ & $\begin{array}{l}14 \text { days after } \\
\text { starting } \\
\text { pravastatin }\end{array}$ & $\begin{array}{l}6 \text { months } \\
\text { after starting } \\
\text { pravastatin }\end{array}$ & $\begin{array}{l}10 \text { months } \\
\text { after starting } \\
\text { pravastatin }\end{array}$ & $\begin{array}{l}18 \text { months } \\
\text { after starting } \\
\text { pravastatin }\end{array}$ \\
\hline $\begin{array}{l}\text { Total Bilirubin (3.4-20.5 } \\
\text { micromol/L) }\end{array}$ & 7.5 & 90.1 & 32.1 & 11 & 8.4 & 10 & 7 & 6 \\
\hline $\begin{array}{l}\text { Direct Bilirubin (0.0-8.6 } \\
\text { micromol/L) }\end{array}$ & - & 60.3 & 21.6 & - & - & - & - & \\
\hline $\begin{array}{l}\text { ALT (Alanine } \\
\text { Aminotransferase, } \\
\text { Normal = 0-55 U/L) }\end{array}$ & 26 & 3756 & 211 & 17 & 18 & 22 & 22 & 18 \\
\hline $\begin{array}{l}\text { AST (Aspartate } \\
\text { Aminotransferase, } \\
\text { Normal = 5-34 U/L) }\end{array}$ & 23 & 3172 & 88 & 25 & 19 & 23 & 23 & 18 \\
\hline $\begin{array}{l}\text { Alkaline Phosphatase } \\
\text { (40-150 U/L) }\end{array}$ & 95 & 397 & 121 & 74 & 77 & 71 & 73 & 71 \\
\hline
\end{tabular}

TABLE 2: Liver function before and after stopping atorvastatin

This illustrates the safety profile of pravastatin in patients who require statins for high LDL management but develop hepatic side-effects on atorvastatin.

\section{Discussion}

Drug-induced liver injury is categorized into three phenotypes based on initial R-value; hepatocellular (Rvalue $>5$ ), cholestatic $(R<2)$, and mixed $(R>2$ but $<5)$. R-value is calculated by the ratio of serum ALT to serum alkaline phosphatase. Our patient had initial $\mathrm{R}$-value $>5$ that attributed her to the hepatocellular group [5]. The Roussel-Uclaf causality assessment method (RUCAM) score is used to estimate the probability that hepatotoxicity has been caused by the drug based on the type of liver injury, timing of onset, assessment of risk factors, course of the reaction, exclusion of other causes, and response to readministration. A score of 0 or less excludes drug-related causes. A score of 1-2 Is characterized as "unlikely," 3-5 as "possible," 6-8 as "probable," while 9 and above is labeled as "highly probable" drugrelated liver injury [6]. Based on our patient characteristics, the patient was found to be in the "highly probable" category. A subset of DILI named "autoimmune-like DILI" has been identified and is difficult to differentiate from "autoimmune hepatitis (AIH)" due to the considerable overlap of clinical and laboratory features [7]. In the event of autoimmune-like DILI, ALT and AST return to normal soon after the discontinuation of the offending agent in most cases without the requirement for immunosuppressants. Furthermore, if AIH is misdiagnosed as immune-mediated DILI and immunosuppressants are used to manage it, there will be a relapse of AIH on cessation of immunosuppressants but DILI almost never relapses after the stoppage of the offending agent [8]. Liver biopsy is not mandatory to make a diagnosis of DILI and is mainly used to rule out other causes [9]. Russo et al. reported 12 (out of a total of 22) cases of statininduced liver injury of hepatocellular phenotype, out of which six had autoimmune-like DILI with $67 \%$ of the cases having ANA and ASMA levels > 1:40 [5]. In our patient, ANAs were weakly positive and so was ASMA with 1:640 titers (that later decreased to 1:80 titers after the discontinuation of atorvastatin) showing autoimmune-like DILI.

The latency period of elevation in aminotransferases after statin initiation is variable, with most of them occurring within 12 weeks [10]. However, latency periods of as long as one year have been observed as well [5]. NICE guidelines recommend baseline LFTs testing before starting statins, then within three months and at 12 months followed by testing only if the patient develops any symptoms related to statins side-effects [11]. American College of Cardiovascular Administrators (ACCA)/American Hospital Association (AHA) guidelines recommend measuring baseline LFTs followed by testing only if clinically indicated [12]. Based on our experience with this case, we recommend the routine follow-up testing of LFTs in asymptomatic patients at least in the initial few months when the risk of hepatotoxicity is highest.

Restarting statin after an episode of liver toxicity is discouraged unless clinically necessary and no other suitable alternative is available [9]. There is limited data on the risk of cross-toxicity between different statins in cases of statin-induced liver injury. However, Liu et al. reported two cases of atorvastatin-induced liver injury who were switched to pravastatin after the return of transaminases to baseline. In both cases, no significant elevation in transaminases from baseline was observed on follow-up [13]. In our patient as well, the use of pravastatin for dyslipidemia management was found to be safe and no derangement of LFTs was observed after 18 months of follow-up, endorsing the possibility that pravastatin has little risk of cross- 
toxicity with atorvastatin and can be safely instituted if clinically indicated in patients who had DILI with atorvastatin.

\section{Conclusions}

Statins-induced liver injury can present in the hepatocellular, cholestatic, or mixed pattern. A subset of liver injury caused by statins called "autoimmune-like drug-induced liver injury" can mimic autoimmune hepatitis. It can be differentiated from autoimmune hepatitis by the normalization of aspartate aminotransferase and alanine aminotransferase levels after the withdrawal of statins therapy. As most cases of statins-induced liver injuries occur early during the initiation of statins therapy, liver function tests should be routinely monitored in the initial few months after starting statins. Furthermore, if a patient develops liver injury due to atorvastatin use, pravastatin has shown a reduced risk of hepatotoxicity in such patients and may be safely used after the normalization of liver enzymes if clinically indicated.

\section{Additional Information \\ Disclosures}

Human subjects: Consent was obtained by all participants in this study. Conflicts of interest: In compliance with the ICMJE uniform disclosure form, all authors declare the following: Payment/services info: All authors have declared that no financial support was received from any organization for the submitted work. Financial relationships: All authors have declared that they have no financial relationships at present or within the previous three years with any organizations that might have an interest in the submitted work. Other relationships: All authors have declared that there are no other relationships or activities that could appear to have influenced the submitted work.

\section{References}

1. Chalasani N: Statins and hepatotoxicity: focus on patients with fatty liver . Hepatology. 2005, 41:690-695. 10.1002/hep.20671

2. Chitturi S, George J: Hepatotoxicity of commonly used drugs: nonsteroidal anti-inflammatory drugs, antihypertensives, antidiabetic agents, anticonvulsants, lipid-lowering agents, psychotropic drugs. Semin Liver Dis. 2002, 22:169-183. 10.1055/s-2002-30102

3. Tolman KG: The liver and lovastatin. Am J Cardiol. 2002, 89:1374-1380. 10.1016/s0002-9149(02)02355-X

4. Jose J: Statins and its hepatic effects: newer data, implications, and changing recommendations. J Pharm Bioallied Sci. 2016, 8:23-28. 10.4103/0975-7406.171699

5. Russo MW, Hoofnagle JH, Gu J, et al.: Spectrum of statin hepatotoxicity: experience of the drug-induced liver injury network. Hepatology. 2014, 60:679-686. 10.1002/hep.27157

6. Danan G, Benichou C: Causality assessment of adverse reactions to drugs-I. A novel method based on the conclusions of international consensus meetings: application to drug-induced liver injuries. J Clin Epidemiol. 1993, 46:1323-1330. 10.1016/0895-4356(93)90101-6

7. Sebode M, Schulz L, Lohse AW: "Autoimmune(-like)" drug and herb induced liver injury: new insights into molecular pathogenesis. Int J Mol Sci. 2017, 18:1954. 10.3390/ijms18091954

8. van Gerven NM, Verwer BJ, Witte BI, et al.: Relapse is almost universal after withdrawal of immunosuppressive medication in patients with autoimmune hepatitis in remission. J Hepatol. 2013, 58:141-147. 10.1016/j.jhep.2012.09.009

9. Chalasani NP, Hayashi PH, Bonkovsky HL, Navarro VJ, Lee WM, Fontana RJ: ACG Clinical Guideline: the diagnosis and management of idiosyncratic drug-induced liver injury. Am J Gastroenterol. 2014, 109:950966. 10.1038/ajg.2014.131

10. Kim H-S, Lee SH, Kim H, et al.: Statin-related aminotransferase elevation according to baseline aminotransferases level in real practice in Korea. J Clin Pharm Ther. 2016, 41:266-272. 10.1111/jcpt.12377

11. National Institute for Health and Care Excellence. Cardiovascular disease: risk assessment and reduction, including lipid modification. (2016). https://www.nice.org.uk/guidance/cg181.

12. Stone NJ, Robinson JG, Lichtenstein AH, et al.: 2013 ACC/AHA guideline on the treatment of blood cholesterol to reduce atherosclerotic cardiovascular risk in adults. A report of the American College of Cardiology/American Heart Association Task Force on practice guidelines. Circulation. 2014, 129:1-45. 10.1161/01.cir.0000437738.63853.7a

13. Liu Y, Cheng Z, Ding L, Fang F, Cheng KA, Fang Q, Shi GP: Atorvastatin-induced acute elevation of hepatic enzymes and the absence of cross-toxicity of pravastatin. Int J Clin Pharmacol Ther. 2010, 48:798-802. $10.5414 /$ cpp 48798 